\title{
Rhotrix polynomials and polynomial rhotrices
}

\author{
S. M. Tudunkaya
}

Kano University of Science and Technology, Wudil, Nigeria

Email address:

tudunkayaunique@yahoo.com (S. M. Tudunkaya)

\section{To cite this article:}

S. M. Tudunkaya. Rhotrix Polynomials and Polynomial Rhotrices, Pure and AppliedMathematics Journal. Vol. 2, No. 1, 2013 , pp. $38-41$. doi: $10.11648 /$ j.pamj.20130201.16

\begin{abstract}
In this piece of note, polynomials defined over the ring $R$ of rhotrices of $n$-dimension and rhotrices defined over polynomials in $\Re$ were explored, the aim is to study their nature and present their properties. The hope is that these polynomials (or these rhotrices) will have wider applications than those polynomials defined over the non-commutative ring of $n$-square matrices (or those matrices defined over polynomials) since $R$ is a commutative ring. The shortcomings of these polynomials and rhotrices were also confirmed as it was proved that the rings $R[x]$ and $R[f]$ are not integral domains.
\end{abstract}

Keywords: Rhotrix, Group, Ring, Polynomial, Commutative Ring, Integral Domain, Mathematical Modeling

\section{Introduction}

In mathematical modeling, real life problems are modeled into polynomial equations from where the coefficient matrices are normally formed and used. The study and analysis of the solutions of these polynomials are used to solve these problems. There is no doubt, exploring polynomials enhances substantive application of Mathematics. The purpose of this note is to define polynomials over a relatively new structure termed rhotrix introduced a decade ago and to define rhotrices over polynomials defined in 9 . These polynomials (or rhotrices) are expected to have more areas of application than the polynomials defined over the non-commutative ring of $n$-square matrices (or matrices defined over polynomials) since the ring $R$ of all rhotrices of $n$-dimension is a commutative ring.

The first work on rhotrices was presented in [1], objects in the set

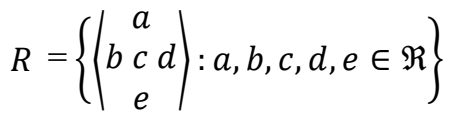

were defined as rhotrices, as a result of their rhomboid nature. The central entry denoted by $h(R)$, was defined as heart (that is $c$ in the above definition). The addition of two rhotrices $R$ and $Q$ was defined as

$$
R+Q=\left|\begin{array}{c}
a \\
b h(R) d \\
e
\end{array}\right|+\left|\begin{array}{c}
f \\
g h(Q) j \\
k
\end{array}\right|
$$

$$
=\left|\begin{array}{c}
a+f \\
b+g(R)+h(Q) d+j \\
e+k
\end{array}\right|
$$

and $-A$ was given as the additive inverse of the rhotrix $A$, for the fact that

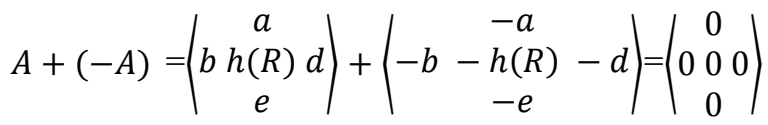

the additive identity of $R$. It was shown that $\{R,+\} \bigcup 0$

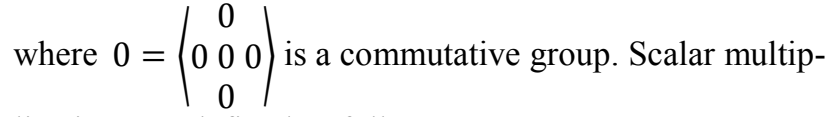
lication was defined as follows:

$$
\alpha R=\alpha\left\langle\begin{array}{c}
a \\
b h(R) d \\
e
\end{array}\right\rangle=\left\langle\begin{array}{c}
\alpha a \\
\alpha b \alpha h(R) \alpha d \\
\alpha e
\end{array}\right\rangle
$$

Multiplication of two Rhotrices $R$ and $Q$ is done as follows:

$$
\begin{gathered}
R \cdot Q=\left|\begin{array}{c}
a \\
b h(R) d \\
e \\
a h(Q)+f h(R) \\
k
\end{array}\right| \cdot\left|\begin{array}{c}
f \\
g h(Q) j \\
k
\end{array}\right|= \\
\left|\begin{array}{c}
b h(Q)+g h(R) h(R) h(Q) d h(Q)+j h(R) \\
e h(Q)+k h(R)
\end{array}\right|
\end{gathered}
$$

It was proved that the set $R$ is a commutative algebra.

$$
\left.I=\mid \begin{array}{ccc}
0 \\
0 & 1 & 0 \\
0
\end{array}\right)
$$


is the multiplicative identity of $R$.

If

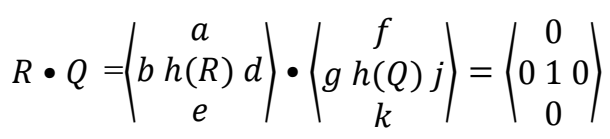

Then

$$
Q=R-1=-\frac{1}{h(R)^{2}}\left|\begin{array}{c}
a \\
b-h(R) d \\
e
\end{array}\right|, h(R) \neq 0
$$

Definition of $R$ was later generalised to dimension $n$ In Mohammed [2], with the same operations defined above as the set

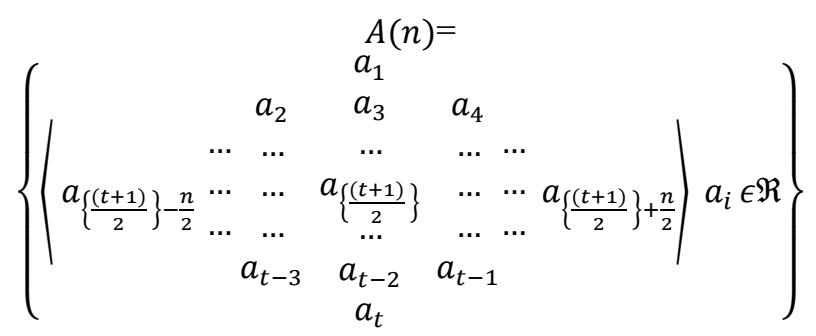

where $t=\frac{\left(n^{2}+1\right)}{2}, n \epsilon 2 Z^{+}+1$ and $\frac{n}{2}$ is the integer value upon division of $n$ by 2 . Hence forth, $\alpha=\left\{\frac{(t+1)}{2}\right\}-\frac{n}{2}$, $\beta=\left\{\frac{(t+1)}{2}\right\}, \pi=\left\{\frac{(t+1)}{2}\right\}+\frac{n}{2}$. In [3], $R$ was classified as a ring and as a field under some conditions even though later it was confirmed that $R$ under the operations defined in [1] can never be an integral domain hence can't be a field in [4].

The main contributions of this work were given in sections 2 and 3. Definitions, results and discussions were presented as according to ideas provided in $[5,6-9,10]$.

\section{Rhotrix Polynomials}

\subsection{Definition}

A polynomial in the indeterminate $x$, over $R$ is an expression of the form

$$
\begin{gathered}
f(x)=A_{0}+A_{1} x+\cdots+A_{m-1} x^{m-1}+A_{m} x^{m} \\
=\sum_{i=0}^{n} A_{i} x^{i}
\end{gathered}
$$

where $A_{i} \in R, m$ a positive integer and $x$ is not found in $R$. $f$ will be termed as rhotrix polynomial.

The set of all polynomials of this form will be denoted by $R[x]$.

\subsection{Definition}

$m$ is the degree of $f$, if $A_{m} \neq 0$ (the zero of $R$ ).

\subsection{Definition}

$A_{m}$ is called the leading coefficient of $f$.

\subsection{Definition}

If

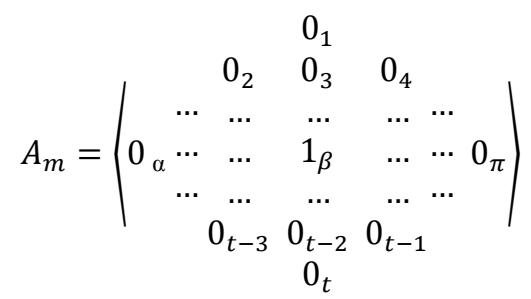

then $f$ monic.

\subsection{Definition}

Define

$$
f=\mid \begin{array}{cccccc} 
& & 0_{1} & & & \\
& 0_{2} & 0_{3} & 0_{4} & & \\
\cdots & \cdots & \cdots & \cdots & \cdots & \\
0_{\alpha} \cdots & \cdots & 0_{\beta} & \cdots & \cdots & 0_{\pi} \\
\cdots & \cdots & \cdots & \cdots & \cdots & \\
& 0_{t-3} & 0_{t-2} & 0_{t-1} & & \\
& & & \\
& & & &
\end{array}
$$

as the zero polynomial.

\subsection{Definition}

Two polynomials

$$
f(x)
$$

And

$g(x)=B_{0}+B_{1} x+\cdots+B_{m-1} x^{m-1}+B_{m} x^{m}=\sum_{i=0}^{n} B_{i} x^{i}$

over

$R$

are said to be equal only if $A_{i}=B_{i}$ for all $i$.

Now by the usual addition and multiplication of polynomials if

$$
f=\sum_{i=0}^{n} A_{i} x^{i}
$$

And

$$
g=\sum_{i=0}^{m} B_{i} x^{i}
$$

Then

$$
f+g=\sum_{i=0}^{\max (n, m)}\left(A_{i}+B_{i}\right) x^{i}
$$

And

$$
f g=\sum_{k=0}^{n+m} C_{k} x^{k}
$$


Where

$$
C_{k}=\sum_{i+j=k} A_{i} B_{j}
$$

The operation of the coefficients is that defined on the commutative the ring $R$.

\subsection{Definition}

If $f(z)=A_{0}+A_{1} z+\cdots+A_{m-1} z^{m-1}+A_{m} z^{m=0}$, then $z$ is called the root of $f$.

With these, the following results will follow:

\subsection{Theorem}

$(R[x],+)$ is a commutative group.

Proof:

The additive identity is the zero polynomial and the additive inverse of

$$
f=\sum_{i=0}^{n} A_{i} x^{i} \text { is }-\sum_{i=0}^{n} A_{i} x^{i}=-f
$$

\subsection{Theorem}

$(R[x], \bullet)$ is a semi group.

Proof:

This follows from the fact that $R$ is also a semi group under the multiplication of rhotrices.

\subsection{Theorem}

$(R[x],+, \bullet)$ is a ring.

Proof:

This follows from 2.7, 2.8 and the distributivity of $R$. The multiplicative inverse of the polynomial $f$ is $g$ such that $f g=1$.

\subsection{Theorem}

$(R[x],+, \bullet)$ is a commutative ring.

Proof:

This follows from the commutativity of $R$.

\subsection{Theorem}

$(R[x],+, \bullet)$ is not an integral domain.

Proof:

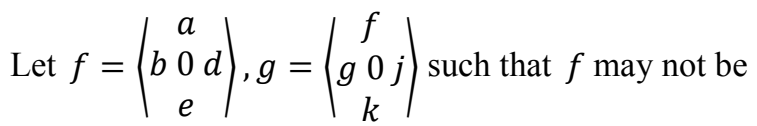
equal to $g \neq 0 \in R$. Also let $c=\left|\begin{array}{c}l \\ m 0 \\ p\end{array}\right| \neq 0 \in R$.

But $c f=c g=0 \in R$ and $f \neq g$.

\section{Polynomial Rhotrices}

Observe that by scalar multiplication of rhotrices,

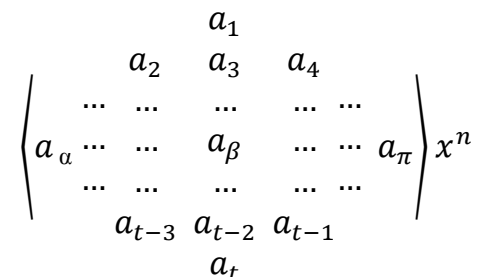

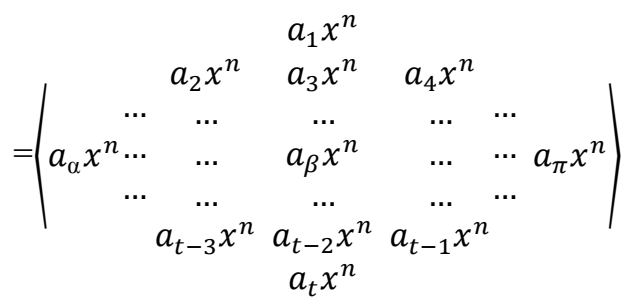

This made the entries of the above rhotrix to be either terms of polynomials or polynomials themselves.

In general, the rhotrix above is better be represented as follows:

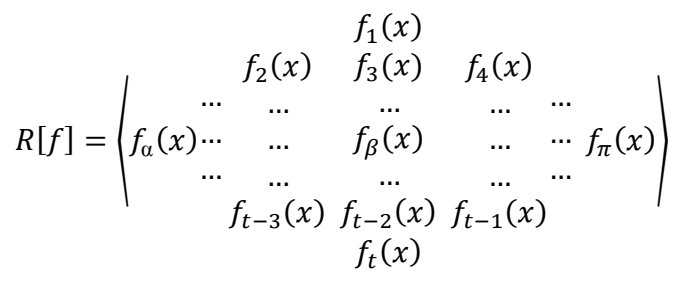

\subsection{Definition}

$R[f]$ will be called polynomial rhotrix.

\subsection{Theorem}

$(R[f],+)$ is a commutative group.

Proof:

The additive identity is the zero of $R$ and the additive inverse of

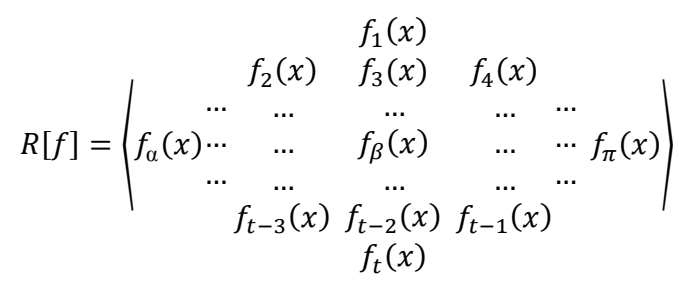

is

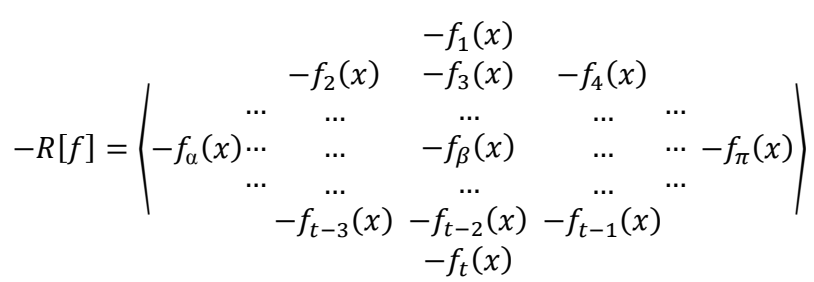

\subsection{Theorem}

$(R[f], \bullet)$ is a semi group.

Proof:

This also follows from the fact $R$ is also a semi group 
under the multiplication of rhotrices.

\subsection{Theorem}

$(R[f],+, \bullet)$ is a ring.

Proof:

This also follows from the fact that $R$ is distributive. Here, the multiplicative inverse of

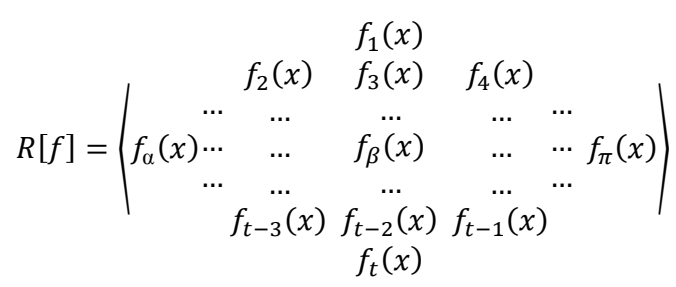

is

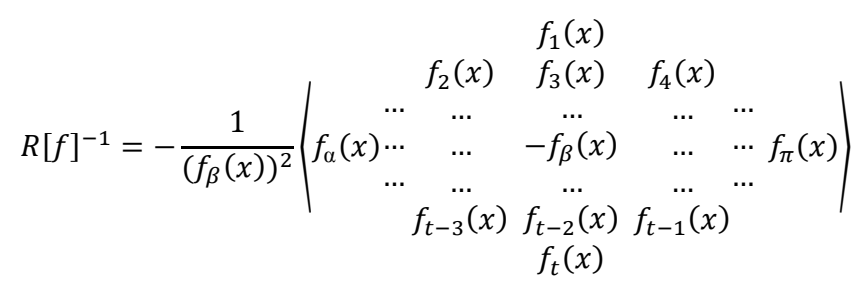

Such that $-f_{\beta}(x) \neq 0$.

\subsection{Theorem}

$(R[f],+, \bullet)$ is a commutative ring.

Proof:

This follows from the commutativity of $R$.

\subsection{Theorem}

$(R[x],+, \bullet)$ is not an integral domain.

Proof:

To prove this theorem, see 2.12 above.

\section{Conclusion}

In this paper, properties of polynomials defined over rhotrices and those of rhotrices defined over polynomials were discussed. The hope is that the presentation of these new structures will pave more ways for further researches and applications of Mathematics in finding solutions to real life problems.

\section{References}

[1] A.O. Ajibade, "The Concept of Rhotrix in Mathematical Enrichment", Int. J. Math. Educ. Sci. Technol., vol. 34 pp. 175-179, 2003.

[2] A. Mohammed, "Theoretical Development and Application of Rhotrices", PhD Dissertation ABU Zaria. Amazon.com, 2011.

[3] A. Mohammed, "A Remark on the Classification of Rhotrices as Abstract Structure", International Journal of Physical Science, vol. 4(9) pp. 496-499, 2009.

[4] S. M. Tudunkaya and S. O. Makanjuola, "Certain Quadratic Extensions". Journal of the Nigerian Association of Mathematical Physics, vol. 22, July issue, 2012.

[5] S. M. Tudunkaya and S. O. Makanjuola, "Certain Constrruction of Finite Fields", Journal of the Nigerian Association of Mathematical Physics, vol. 22, November issue, 2012.

[6] B. Cherowitz, "Introduction to finite fields". (http://www.cudenver.edu/echeroni/vboutdrd/tin_lds.html), 2006.

[7] S. Lang, Algebra: Graduate Texts in Mathematics (fourth edition), New York, Springer-Verlag, 2004.

[8] L. R. Jaisingh, Abstract Algebra (second edition). McGRAW-HILL, New York, 2004.

[9] E.,Brent, Symmetries of equation: An introduction to Galois theory: University of York, York Y010 5DD, England, 2009.

[10] S. M. Tudunkaya, and S. O. Makanjuola, "Note on Classification of Rhotrices as Abstract Structure", unpublished. 